CAHIERS DE

NARRATOLOGIE

\section{Cahiers de Narratologie}

Analyse et théorie narratives

17 | 2009

Stéréotype et narration littéraire

\title{
La Sémantique des Possibles Argumentatifs : un modèle de description-construction-représentation des significations lexicales
}

Virginie Marie

\section{(2) OpenEdition}

Journals

Édition électronique

URL : http://journals.openedition.org/narratologie/1337

DOI : $10.4000 /$ narratologie. 1337

ISSN : 1765-307X

Éditeur

LIRCES

Référence électronique

Virginie Marie, «La Sémantique des Possibles Argumentatifs : un modèle de description-constructionreprésentation des significations lexicales », Cahiers de Narratologie [En ligne], 17 | 2009, mis en ligne le 22 décembre 2009, consulté le 15 novembre 2019. URL : http://journals.openedition.org/narratologie/ 1337 ; DOI : 10.4000/narratologie.1337

Ce document a été généré automatiquement le 15 novembre 2019.

\section{(c) (i) (9)}

Cahiers de Narratologie - Analyse et théorie narratives est mis à disposition selon les termes de la licence Creative Commons Attribution - Pas d'Utilisation Commerciale - Pas de Modification 4.0 International. 


\title{
La Sémantique des Possibles Argumentatifs : un modèle de description-construction- représentation des significations lexicales
}

\author{
Virginie Marie
}

Le stéréotype est une notion contemporaine puisque celle-ci fait son apparition dans le vocabulaire courant seulement vers la fin du XIXe siècle, et intervient dans le domaine des sciences sociales à partir des années 1920. A l'origine, le stéréotype désigne un objet imprimé dupliqué à l'infini grâce à la reproduction d'un modèle ${ }^{1}$. C'est par extension métaphorique que va s'opérer le passage du sens littéral au sens figuré. Le concept sera repris par LIPPMAN W. dans son Public Opinion en 1920. La définition proposée par l'auteur dans le champ des sciences sociales a nourri la réflexion contemporaine. L'imprécision du concept a en quelque sorte garanti sa flexibilité et sa capacité à se redéfinir en fonction des secteurs de la recherche: opinion publique, attitudes et comportements, préjugé, rôle social, interactions en groupes... La redéfinition du stéréotype en termes non seulement d'images mais aussi d'idées reçues contribue ainsi aux recherches sur l'opinion publique, à l'analyse de son fonctionnement et à la dénonciation de ses excès. Dans la mesure où le stéréotype relève d'un processus de catégorisation et de généralisation, il simplifie et élague le réel ; il peut ainsi favoriser une vision schématique et déformée de l'autre qui entraîne des préjugés. C'est dans ce sens que vont, jusqu'à nos jours, de nombreuses tentatives de définition ${ }^{2}$. En nous appuyant sur les diverses définitions apportées par les principaux chercheurs (MORFAUX LM ${ }^{3}$, AMOSSY R., BOUCHE C., HURFORD JR. et HEASLEY B., KLEIBER G.), nous pouvons reprendre ou rappeler de manière exhaustive les traits définitoires attribués au stéréotype en retenant cinq grands critères :

« la fréquence : un stéréotype est une structure souvent répétée ;

le figement : l'emploi répétitif du stéréotype a conduit au figement de l'association 
des termes du stéréotype ;

l'absence d'origine précisément repérable ;

la prégnance dans la mémoire collective : un stéréotype se signale par son caractère durable et son inscription dans la mémoire socioculturelle ;

le caractère abstrait et synthétique: lorsqu'il consiste en une représentation thématique ou idéologique, le stéréotype apparaît comme une formule simplifiée » (MORFAUX, 1980 : 34).

Dans le cadre de notre travail, il nous a semblé pertinent de rappeler brièvement les fondamentaux de la notion afin de pouvoir introduire la sémantique des possibles argumentatifs (désormais SPA) proposée et développée par GALATANU qui se présente comme un modèle de description-construction-représentation des significations lexicales censées rendre compte $d u$ " cinétisme ${ }^{4}$ " des significations. Un modèle qui s'appuie principalement sur des mécanismes sémantico-discursifs et pragmaticodiscursifs d'activation du potentiel argumentatif. Cette description de la signification lexicale repose à la fois sur des informations sémantiques et discursives et participe ainsi à la reconstruction de la signification lexicale d'un mot en s'appuyant sur des hypothèses provenant de l'interprétation du sens et des occurrences du mot.

Dans cet article, nous exposerons les origines de la SPA, ses principes de description de la signification lexicale et ses applications à partir de notions (francophile, francophilie, francophone, francophonie) qui rendront compte des déploiements argumentatifs en analysant l'évolution des définitions lexicographiques étudiées en termes de noyau et de stéréotypes.

I. La Sémantique des Possibles Argumentatifs

Le développement de la SPA s'appuie sur la recherche d'un modèle de représentation de la signification lexicale, ou plus précisément de la reconstruction de la signification lexicale à partir de l'élaboration de certaines hypothèses découlant de l'interprétation du sens des diverses occurrences du mot concerné. Cette interprétation du sens donne à saisir les représentations suivantes :

- « celles du monde « perçu » et « modélisé » par la langue (KLEIBER, 1999 : 27-34);

- celles du « potentiel discursif » au niveau des enchaînements argumentatifs des mots, qui fait l'objet privilégié de la sémantique argumentative, dans ses différents développements (ANSCOMBRE et DUCROT, 1983 ; ANSCOMBRE et DUCROT, 1995 ; CAREL et DUCROT, 1999) et que l'environnement sémantique de la phrase énoncée et/ou le contexte pragmatique de cet énoncé, peuvent activer, voire renforcer, ou au contraire, affaiblir, voire neutraliser (GALATANU, 1999, 2003, 2005, 2006)» (GALATANU, 2007a).

En tenant compte des fondements de la SPA, présentés précédemment, le modèle de représentation sémantique SPA est contraint par quatre conditions qu'il se doit de remplir conjointement.

- Ainsi, cette représentation doit comporter une dimension descriptive de la signification lexicale, préservant ainsi le recours à une lecture de la forme logique de la phrase énoncée ${ }^{5}$;

- faire apparaître à la fois la « partie stable » de la signification et la partie évolutive, que le discours proposé charge et/ou décharge de valeurs ;

- rendre compte du statut d'ensemble ouvert des éléments de signification évolutifs, appelés «stéréotypes";

- rendre compte du potentiel discursif (argumentatif) de la signification lexicale.

Plus précisément, la SPA décrit la signification lexicale d'un mot, lequel comporterait trois strates: le noyau (comprenant les traits de catégorisation sémantique), les 
stéréotypes (l'ensemble d'associations des éléments du noyau avec d'autres représentations), les possibles argumentatifs (au sens de séquences discursives).

D'un point de vue méthodologique, l'analyse sémantique des entités lexicales se traduit par une construction de l'objet théorique à partir d'hypothèses externes sur le sens des occurrences du mot analysé en contexte. Cette étape correspond à la collecte et à l'étude du ou des discours lexicographiques; ou à la collecte et à l'étude d'énoncés produits et/ou relevés dans des séquences de communication. Dans cette perspective, l'analyse des définitions lexicographiques a pour objectif premier l'émergence des éléments stables du noyau de la signification du mot. Une fois l'analyse du noyau achevée, il importe de construire les stéréotypes. De la même manière que le noyau, il s'agit de relever des informations lexicographiques. Enfin, l'ultime étape consiste à mettre en exergue le caractère cinétique des significations lexicales. C'est bien à partir de ces déploiements argumentatifs que l'on pourra reconstruire les significations des mots.

La sémantique des possibles argumentatifs est :

- - « un modèle de représentation du discours comme lieu de manifestation de mécanismes sémantico-discursifs de construction de sens et de re-construction de la signification, mécanismes qui expliquent ce cinétisme entendu comme processus holistique et associatif,

- - un modèle de représentation de la signification linguistique susceptible de rendre compte de sa partie stable et de son noyau » (GALATANU, 2006).

Cette approche permet de restituer la fonction argumentative de la signification du mot et de décrire les entités linguistiques par leur rapport avec les enchaînements argumentatifs du discours qu'elles rendent possibles.

\section{I.1 Noyau et stéréotypes}

Le noyau se compose de traits nécessaires de catégorisation (TNC) et de marques abstraites. Ces TNC apportent les informations suivantes: la nature grammaticale du mot (nom, verbe...), ses caractéristiques modales (aléthique, intellectuelle...) et son orientation axiologique (positive ou négative).

Dans le modèle de la SPA, les stéréotypes coïncident avec la déclinaison des marques (prédicats) abstraites du noyau en interaction avec d'autres représentations sémantiques. Tous ces ensembles forment des blocs d'argumentation internes à la signification lexicale. Un bloc d'argumentation interne représente une association de prédicats appartenant à la signification d'une entité lexicale dans une structure argumentative (CAREL et DUCROT, 1999).

Afin d'illustrer cette présentation du noyau et des stéréotypes, nous reprenons l'exemple de GALATANU qui nous permet d'illustrer la construction discursive de soi et du monde au travers du sens de l'argumentatif. Cette analyse porte sur le mot guerre étudié dans les discours des médias :

«la qualification des différents éléments de sa signification, , peut convoquer des topoï à orientation axiologique positive ou négative. Les modificateurs qui s'organisent autour de la cause défendue par l'un ou l'autre des groupes «belligérants» construisent un monde où la guerre est perçue comme un phénomène moralement et politiquement positif, souhaitable même (les côtés négatifs - la mort, la souffrance - sont occultés) : guerre d'indépendance/juste/de défense, ou au contraire (c'est le cas le plus fréquent de nos jours, où l'on commence à parler de la guerre comme d'une valeur négative, opposée à la valeur « universellement » positive de paix), un monde où la guerre est perçue comme un phénomène moralement et politiquement négatif : guerre raciale/de conquête/ 
injuste ou encore une sale guerre. [...] Le discours ainsi un monde où la guerre, " cette guerre» est "aveugle comme peu de guerre le sont» et «frappe directement les innocents " » (GALATANU, 1999c : 38-39).

Dans l'approche de la sémantique argumentative, nous constatons que la signification lexicale décrit et renvoie au monde. Ce procédé est rendu possible par le stéréotype lié au mot et par les représentations associées aux éléments de ce même stéréotype, qui lui-même, peut aussi à son tour être associé à d'autres stéréotypes.

I.2 La stéréophagie

L'analyse des formes de manifestation des stéréotypes et du processus de stéréotypage se trouve,

«par définition à l'interface de l'Analyse du Discours et de l'Analyse des pratiques sociales et de la Praxéologie, définie comme la science de l'Action Humaine »

(GALATANU, $2006:$ 89-90) :

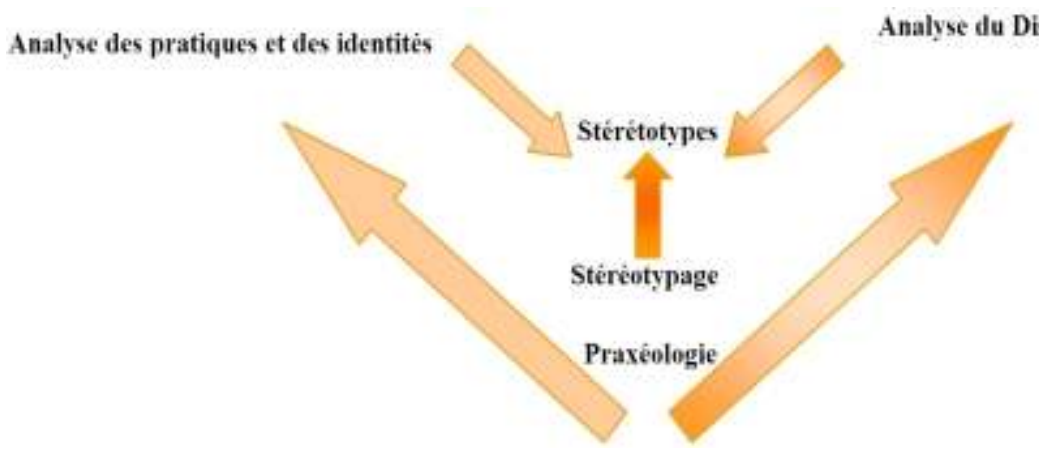

Figure 1 : le stéréotype (GALATANU, 2006 : 90)

Le phénomène discursif appelé "stéréotypage » permet le déploiement de l'un des éléments du noyau jusqu'aux derniers enchaînements, par la proposition de nouveaux stéréotypes. Le concept de stéréotypage est l'une des formes de manifestation du cinétisme de la signification lexicale. Nous comprenons ici par stéréotypage une affiliation de l'un des éléments du noyau avec une autre représentation dans un bloc d'argumentation interne, ou exposée comme appartenant à l'ensemble ouvert des stéréotypes de la signification du mot.

I. 3 Modèle « quantique »

Ce modèle est élaboré à partir de l'interprétation des énoncés menant à la construction, reconstruction, présentation de systèmes de valeurs. Il s'agira donc ici de s'intéresser à :

«l'étude des mécanismes langagiers qui habilitent le discours à être un terrain privilégié d'influence d'autrui, de présentation de soi, de constructions identitaires, de présentation ou de reconstruction d'un système de valeurs »

(GALATANU, 1999a).

Par ailleurs, GALATANU cherche " une description de l'argumentation qui puisse recouvrir aussi bien des phénomènes inférentiels, s'appuyant sur des données contextuelles et/ou proposées par le co-texte, phénomènes qui relèvent, respectivement de la "pragmatique inférentielle» et de la rhétorique, que des phénomènes discursifs proprement linguistiques, s'appuyant sur le potentiel argumentatif des mots et relevant de la pragmatique intégrée ou, dans sa redéfinition récente, de la sémantique argumentative »(idem). Il s'agit bien d'articuler le système argumentatif linguistique avec des phénomènes inférentiels. Pourtant, GALATANU 
montre que la sémantique argumentative ne rend pas compte de certains phénomènes discursifs : "la sémantique argumentative rend compte des enchaînements discursifs d'un énoncé sur la base [...] seulement de ces enchaînements discursifs. Ainsi, l'analyse [ne] rend pas compte des énoncés (iii) et (iii'), le dernier étant bivalent quant aux enchaînements possibles, ni de l'énoncé (iv) qui transgresse les topoï qui autorisent linguistiquement des enchaînements "normaux" (cette transgression est pourtant souvent présente dans les séquences argumentatives au sens traditionnel du terme et permettent de parler de «mauvais arguments»), ni enfin du sens de l'énoncé (v), qui propose un nouveau "topos" (comme c'est également souvent le cas des argumentations séquentielles) :

- (iii) ceci se passe à deux heures de Paris, donc il faut intervenir

- (iii') ceci se passe à deux heures de Paris, enchaînements possibles : c'est loin, ça ne nous regarde pas/c'est tout près ça nous concerne, donc il faut intervenir

- (iv) à force d'être raisonnable, vous allez finir par vous acheter une voiture de luxe

- (v) il est très intelligent, donc il ne peut pas être ordonné (ibidem).

Pour rendre compte alors d'un éventail plus large de phénomènes, le modèle sémantique comporte trois niveaux :

«La signification lexicale est constituée à la fois d'un noyau (par analogie avec le noyau de l'atome): traits dits "nécessaires", de catégorisation, et stéréotype associé au mot et de possibles argumentatifs possibles qui relient des éléments du stéréotype à d'autres représentations sémantiques (stéréotypes d'autres mots) et qui se superposent (comme les états des électrons), dans une vision holistique du sens. Ces possibles argumentatifs nous les avons décrits comme des «nuages topiques " reliant les éléments du stéréotype du mot à d'autres représentations sémantiques. C'est l'interaction avec d'autres significations qui forment (construisent) l'environnement discursif, qu'il soit linguistique ou inférentiel (pragmatique) qui provoque un phénomène de séparation des possibles argumentatifs et stabilisent un sens. Ce phénomène est similaire au phénomène de décohérence qui permet l'articulation du monde quantique et du monde physique qui est le nôtre et dans lequel les objets complexes, formés d'atomes ne présentent pas des états superposés »

(GALATANU, 1999b).

En synthétisant, dans les prémices de la SPA, la signification lexicale est décrite par homologie avec la structure quantique de l'atome. Cette analogie repose sur trois grands principes :

- sur le noyau de la signification lexicale, similaire au noyau de l'atome où l'on trouve les stéréotypes présents dans les structures argumentatives ${ }^{6}$.

- sur la présence de stéréotypes, les traits argumentatifs possibles, équivalents aux électrons gravitant autour du noyau de l'atome.

- sur la présence de PA, reliant des éléments du stéréotype à d'autres représentations sémantiques, semblables à un « nuage topique » (électrons topiques) (GALATANU, 1999a : 49-50).

Les PA se décrivent ainsi en termes de blocs argumentatifs externes. Ils se dotent à la fois d'un déploiement argumentatif possible qui peut être normatif ou transgressif et d'un potentiel axiologique possible avec une polarité (positif/négatif).

Cette structuration en différents niveaux permet de mieux appréhender les nuances modales dans la signification des mots :

«par exemple, la valeur axiologique négative de mots comme crime, viol, vol, fait partie des éléments de leurs stéréotypes, alors que la bivalence ou la polarité des 
mots comme guerre, grève, fait partie des possibles argumentatifs de leurs stéréotypes, et l'un ou l'autre des pôles axiologiques (positifs ou négatifs) va être sélectionné et activé, dans le discours par un processus de contamination avec les stéréotypes des mots de leur environnement, stabilisant ainsi une orientation argumentative : sale guerre, guerre juste, guerre de défense »

(GALATANU, 2000a).

D'un point de vue méthodologique, l'analyse sémantique des entités lexicales se traduit par une construction de l'objet théorique à partir d'hypothèses externes sur le sens des occurrences du mot analysé en contexte. Cette étape correspond à la collecte et à l'étude du ou des discours lexicographiques; ou à la collecte et à l'étude d'énoncés produits et/ou relevés dans des séquences de communication. Dans cette perspective, l'analyse des définitions lexicographiques a pour objectif premier l'émergence des éléments stables du noyau de la signification du mot. Une fois l'analyse du noyau achevée, il importe de construire les stéréotypes. De la même manière que le noyau, il s'agit de relever des informations lexicographiques. Enfin, l'ultime étape consiste à mettre en exergue le caractère cinétique des significations lexicales. C'est bien à partir de ces déploiements argumentatifs que l'on pourra reconstruire les significations des mots.

Nous pouvons illustrer cette approche méthodologique par une étude du mot grâce $\boldsymbol{~}^{7}$ en présentant par exemple le noyau et la déclinaison des possibles argumentatifs. En nous reportant ainsi à la définition donnée par le dictionnaire, Le Nouveau Petit Robert, nous constatons que le mot grâce est polysémique. Nous nous proposons de déterminer le noyau de ce mot en nous appuyant sur les différentes définitions qui rendent compte de cette polysémie. Afin de rendre l'étude plus pertinente nous tenterons d'en donner les traits et de définir le noyau à partir duquel nous déclinerons le bloc argumentatif interne et les stéréotypes qui peuvent lui être rattachés. Pour faciliter la lecture, nous avons fait apparaître les diverses définitions dans un tableau. Celui-ci se divise en deux catégories: grâce non physique et grâce physique. Cette division nous permettra d'établir un classement interne qui rendra plus efficace le repérage des traits et des valeurs du mot grâce.

Grâce non physique

\begin{tabular}{|c|c|c|}
\hline \multirow{3}{*}{$\begin{array}{l}\text { CLASSE } \\
\text { A }\end{array}$} & Contexte 1 & $\begin{array}{l}\text { Objet d'un don, positif, agréable, gratuit (pas forcément mérité). Acte de donner, } \\
\text { agréable (gratuit) } \\
\text { Un don non justifiable. }\end{array}$ \\
\hline & Contexte 2 & $\begin{array}{l}\text { La faveur de Dieu, la grâce de Dieu, œuvre de grâce. } \\
\text { Un don non justifiable. } \\
\text { La faveur (la grâce) dans ce contexte associe l'humain et le divin. }\end{array}$ \\
\hline & Contexte 3 & $\begin{array}{l}\text { Faveur de Dieu avec une toute autre terminologie : le Salut. } \\
\text { Dieu accorde, donne et/ou reprend sa grâce (don non justifiable). } \\
\text { Grâce efficace, suffisante, sanctifiante. } \\
\text { Etre en état de grâce. } \\
\text { divine, on accorde quelque chose. } \\
\text { C'est le sens de la faveur divine chrétienne. } \\
\text { « Faveur divine »: fait intervenir la volonté divine et le salut de l'homme. }\end{array}$ \\
\hline
\end{tabular}




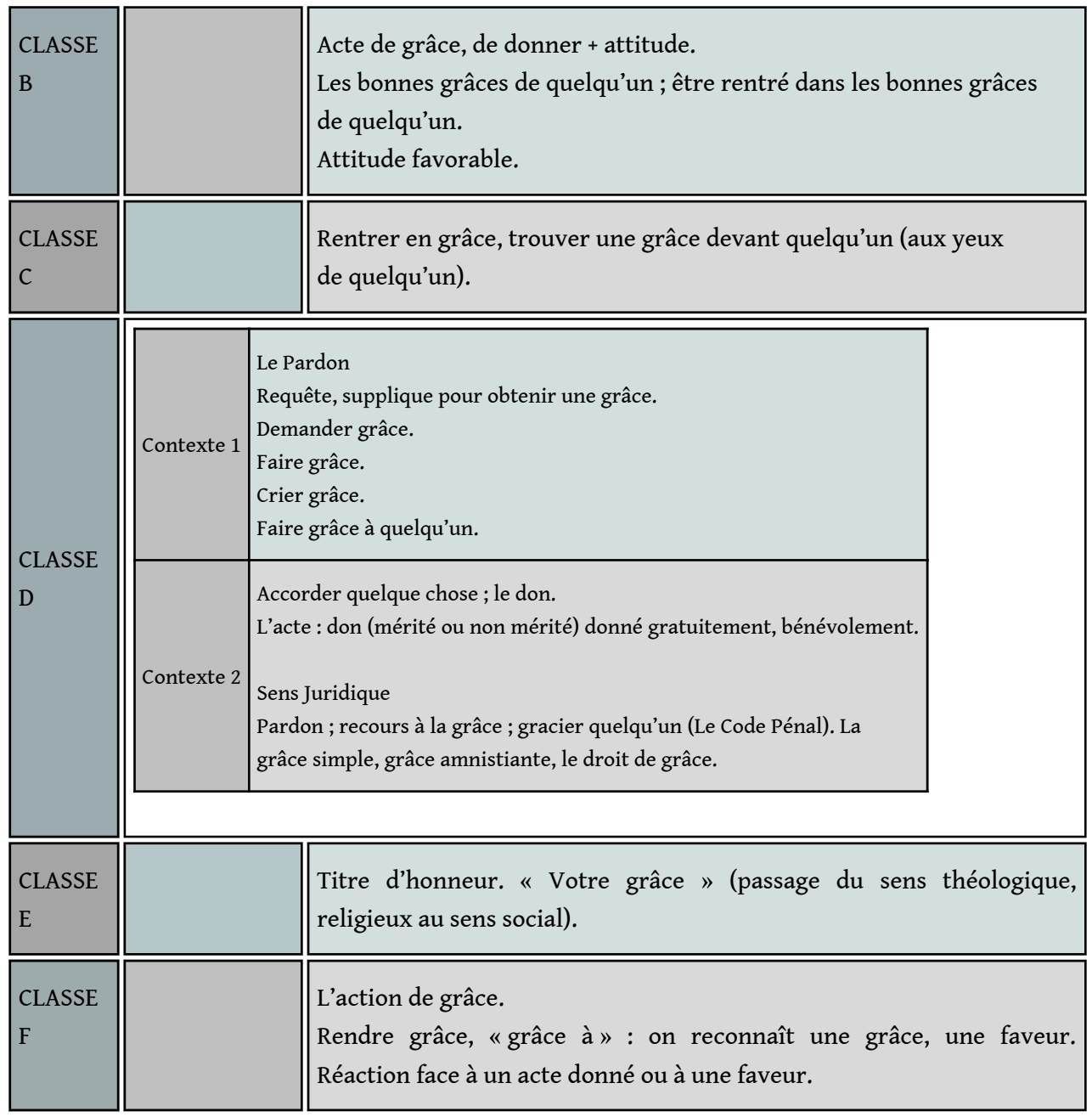

\section{Grâce physique}

\begin{tabular}{|l||l|}
\hline CLASSE A & $\begin{array}{l}\text { Grâce naturelle. } \\
\text { Grâce des gestes, des mouvements. } \\
\text { Avoir de la grâce. } \\
\text { Attrait, charme d'une personne. } \\
\text { S'exprimer avec grâce. } \\
\text { « Les Trois Grâces » : personnification du don de plaire. } \\
\text { Evaluation positive du mot grâce dans ce contexte. }\end{array}$ \\
\hline
\end{tabular}

\section{Figure 1 : L'étude du mot grâce}

\begin{tabular}{|l|l|}
\hline Le noyau dur & Grâce : \\
\hline \hline prédicat nominal & \\
\hline \hline axiologie + (grâce physique) & \\
\hline
\end{tabular}




\begin{tabular}{|l|l|}
\hline affectif et moral & \\
\hline \hline aléthique & \\
\hline \hline caractère positif & \\
\hline \hline donnée & \\
\hline \hline non justifiée & \\
\hline \hline gratuite & \\
\hline \hline naturelle & \\
\hline
\end{tabular}

A partir de ce noyau dur nous pouvons donner une conceptualisation onomasiologique qui s'appuie sur cette double représentation : grâce non physique et grâce physique.

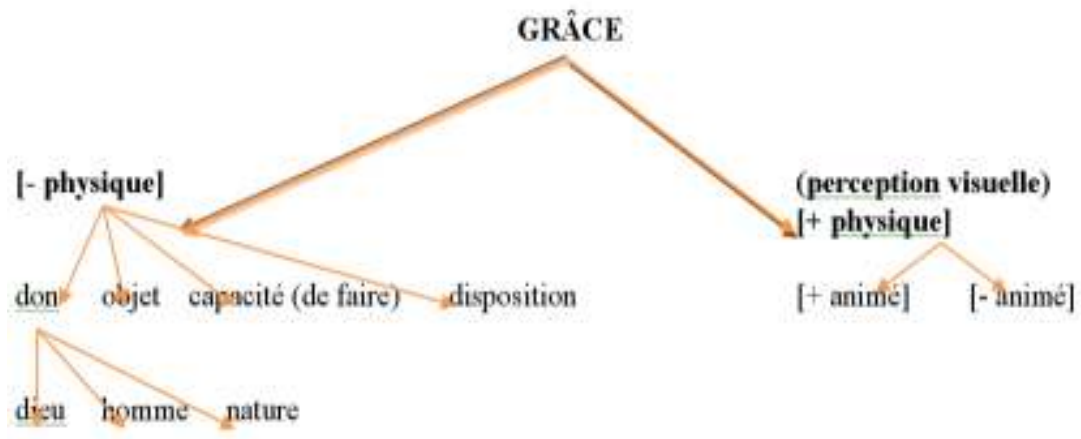

Figure 2 : La conception onomasiologique

2) Déclinaison du bloc argumentatif

- S vouloir être agréable envers $\mathbf{A}^{8}$

- A obtenir pardon de $\mathbf{S}$

- $\mathbf{S}$ avoir don

- S avoir charme

- S avoir bonne intention envers A

3) Les stéréotypes

- $\mathbf{S}$ vouloir être agréable envers A

- donc $\mathbf{S}$ vouloir faire du bien à $\mathbf{A}$

- donc $\mathbf{S}$ faire preuve de bonté envers $\mathbf{A}$

- donc $\mathbf{S}$ vouloir faire plaisir à $\mathbf{A}$

- donc $\mathbf{S}$ aider $\mathbf{A}$

- donc $\mathbf{A}$ demander quelque chose à $\mathbf{S}$

- donc A mériter faveur de $\mathbf{S}$

- donc A éprouve satisfaction

- A obtenir pardon de $\mathbf{S}$

- donc A a mal agi donc A mauvais

- donc $\mathbf{S}$ accorder pardon donc $\mathbf{S}$ bon 
- donc S épargner A donc A sauver

- donc A reconnaissant envers $\mathbf{S}$

- donc A remercier (ou louer) S

- S avoir don

- donc S penser être Bien

- donc $\mathbf{S}$ avoir des qualités

- donc S penser être exceptionnel

- donc $\mathbf{S}$ est heureux

- donc S accomplit des choses

- S avoir charme

- donc $\mathbf{S}$ posséder attraits

- donc S être beau

- donc S plaire

- donc $\mathbf{S}$ se sentir satisfait

- S avoir bonne intention envers A

- donc S faire preuve de bonne volonté envers A

- donc $\mathbf{S}$ aimable avec $\mathbf{A}$

- donc A apprécier $\mathbf{S}$

- donc $\mathbf{S}$ vouloir faire du bien à $\mathbf{A}$

- donc $\mathbf{S}$ vouloir faire plaisir à $\mathbf{A}$

- donc $\mathbf{S}$ aider (ou donner) avec plaisir à $\mathbf{A}$

- donc A recevoir gratuitement de $\mathbf{S}$

Le stéréotype résulte d'un mécanisme complexe comme nous le montre l'exemple cidessus.

I.4 Le noyau sémantique

En suivant le modèle ci-dessus, nous avons répertorié les diverses définitions données aux mots «Francophile, Francophilie, Francophone, Francophonie» par les dictionnaires de langue et de spécialité, il semble pertinent d'extraire un sens minimal de chacune de ces notions. Pour cela, nous nous appuyons sur la Théorie des blocs argumentatifs et la Théorie des topoï, telles que les conçoivent DUCROT, O., CAREL, M., ANSCOMBRE, JC. Et GALATANU O. en partant du principe que l'argumentation réside dans la signification du mot. Cette signification minimale, appelée noyau sémantique, est donc exprimable avec des entités abstraites articulées dans une construction logique qui serait exprimée à l'aide des connecteurs donc et pourtant.

Pour Francophile, le noyau peut être représenté comme ceci :

S AIMER PARLER FRANÇAIS DONC SE SENTIR PROCHE DES FRANÇAIS

ET

S AIMER LA FRANCE DONC SE SENTIR PROCHE DE LA COMMUNAUTE FRANCAISE

Pour Francophilie :

S AIMER LA FRANCE DONC S FAVORABLE

Pour Francophone :

S PARLER FRANÇAIS DONC S APPARTENIR A UN ESPACE

Pour Francophonie, nous reprenons les travaux de GALATANU O. (2008a) :

$S$ SAVOIR DONC POUVOIR PARLER FRANÇAIS

ET

S DEVOIR PARLER FRANÇAIS

ET

S APPARTENIR A UNE COMMUNAUTE 
Cette dernière notion (francophonie) paraît plus difficile à exprimer en termes de noyau sémantique car elle renvoie à un grand nombre de réalisations. Les expressions qui viennent d'être adoptées traduisent l'idée de l'individu ou d'un ensemble d'individu qui poserait l'existence d'une communauté dans laquelle il se reconnaîtrait.

Déterminer le noyau sémantique de ces mots permet de s'assurer que les expressions formées avec ces mots peuvent être renvoyées à cette signification minimale et en représenter des associations stéréotypiques qui peuvent produire des orientations à partir de possibles argumentatifs. Nous avons donc tenté de rendre compte de l'existence des associations stéréotypiques et $\mathrm{du}$ déploiement des possibles argumentatifs pour les mêmes concepts :

\section{Francophile}

\begin{tabular}{|l|l|}
\hline Noyau & Possibles Argumentatifs \\
\hline \hline S aimer une communauté & donc S se sentir proche \\
\cline { 2 - 2 } & donc S s'intéresser à la culture de cette communauté \\
\cline { 2 - 2 } & donc S s'intéresser à la langue \\
\hline & donc $S$ défendre le pays \\
\hline
\end{tabular}

\section{Francophilie}

\begin{tabular}{|l||l|}
\hline Noyau & Possibles Argumentatifs \\
\hline \hline S aimer la France & donc S favorable \\
\cline { 2 - 2 } & donc S vouloir partager \\
\hline & donc S vouloir connaître \\
\hline
\end{tabular}

\section{Francophone}

\begin{tabular}{|l|l|}
\hline Noyau & Possibles Argumentatifs \\
\hline \hline S savoir parler français & donc $\mathrm{S}$ appartenir à un espace \\
\cline { 2 - 2 } & donc $\mathrm{S}$ partager les valeurs \\
\hline & donc $\mathrm{S}$ avoir des compétences linguistiques \\
\hline
\end{tabular}


POUR Francophonie,

Nous nous appuyons sur les travaux de GALATANU O. (2008a) :

\begin{tabular}{|l|l|}
\hline Noyau & Possibles Argumentatifs \\
\hline \hline S savoir & donc S pouvoir parler français \\
\cline { 2 - 2 } & donc S pouvoir communiquer \\
\hline \hline S devoir parler français & donc S développer des facultés \\
\hline \hline S appartenir à une communauté & donc parler français \\
\hline
\end{tabular}

En nous référons aux discours lexicographiques des dictionnaires, nous voyons apparaître quelques éléments stables, que nous retrouvons dans l'étymologie du mot " francophonie ", que l'on pourrait résumer comme suit : "état de celui qui parle la langue française/d'une communauté humaine qui utilise la langue française dans l'essentiel des pratiques sociales» (GALATANU : 2008a). De cette observation, nous pouvons proposer, dans le cadre de la SPA, une signification construite à partir des discours lexicographiques :

FRANCOPHONIE

\begin{tabular}{|c|c|}
\hline Noyau & Stéréotypes \\
\hline \multirow{2}{*}{$\begin{array}{l}\text { S SAVOIR DC POUVOIR PARLER FRANÇAIS } \\
\text { (E1) }\end{array}$} & E1 DC compétence native \\
\hline & E1 DC compétence apprise \\
\hline \multicolumn{2}{|l|}{ Et } \\
\hline \multirow[t]{3}{*}{ S DEVOIR PARLER FRANÇAIS (E2) } & E2 DC parler français habituellement \\
\hline & E2 DC parler français fréquemment \\
\hline & $\begin{array}{l}\text { E2 DC parler français dans la communication } \\
\text { institutionnelle }\end{array}$ \\
\hline
\end{tabular}

(GALATANU : 2008a)

Dans l'approche de la SPA, l'analyse sémantique des entités lexicales est appréhendée essentiellement comme une construction de l'objet étudié en formulant des hypothèses externes sur le sens des diverses occurrences répertoriées. Cette première démarche (ou opération) correspond à l'étude des discours lexicographique(s) sélectionnés mais aussi, ultérieurement, aux énoncés produits dans notre corpus. Ce qui nous amène à 
évoquer la deuxième démarche qui consiste à repérer des occurrences dans un discours bien défini en tenant compte de certains critères praxéologiques (par exemple, dans notre cas, il s'agit de textes institutionnels et de textes recueillis auprès de différents publics portant sur la Francophonie/la Francophilie. Plus exactement, l'environnement discursif influe sur la signification du mot, et cela, à travers les déploiements discursifs ou argumentatifs, et cette contamination semble d'autant plus jouer un rôle important dans la construction des possibles argumentatifs, puisqu'elle a cette capacité à accepter ou à réfuter les déploiements obtenus à partir du dispositif stéréotypique. Par ailleurs, en reprenant les propos de GALATANU :

«c'est à partir de ces déploiements discursifs du potentiel argumentatif du mot que l'on peut reconstruire une signification dont le caractère évolutif et le cinétisme ont été posés d'emblée comme des à priori de l'approche sémantique » (GALATANU : 2008a).

II. Analyse du concept « Francophonie » dans les discours lexicographiques et dans le processus de dénomination institutionnelle

Le concept de francophonie est une notion complexe et connaît de nombreuses évolutions. De ce fait, les définitions institutionnelles, politiques sont abondantes et réfèrent à des faits appropriés, mais elles semblent s'éloigner du noyau de la signification du mot proposée par les discours lexicographiques (TLF, Robert, Larousse) que nous pouvons formuler de la manière suivante, en reprenant ici un article de GALATANU (2008a : 6-8), qui nous présente l'évolution du noyau Francophonie :

$$
\text { Noyau : APPARTENIR A LA COMMUNAUTE X donc PARLER FRANÇAIS }
$$

GALATANU postule que des stéréotypes sémantiques pourront s'organiser autour des quatre axes définis par DENIAU (DENIAU, 1983): linguistique, spirituel et institutionnel, déclinant les éléments du noyau dans les blocs d'argumentation interne :

\begin{tabular}{|c|c|}
\hline \multirow[t]{5}{*}{ Stéréotypes: } & APPARTENIR A LA COMMUNAUTE X donc HABITER LA REGION X \\
\hline & $\begin{array}{l}\text { APPARTENIR A LA COMMUNAUTE X dONC VIVRE SELON TEL SYSTEME DE NORMES SOCIALES/ } \\
\text { MORALES/CODES DE CONDUITE }\end{array}$ \\
\hline & PARLER FRANÇAIS donc UTILISER TEL LEXIQUE/SYNTAXE \\
\hline & APPARTENIR A LA COMMUNAUTE X donc PARTAGER UN ESPACE \\
\hline & $\begin{array}{l}\text { INSTITUTIONNEL/PARTAGER TEL SYSTEME DE VALEURS/AGIR DE TELLE MANIERE/ETRE } \\
\text { SOLIDAIRE DES AUTRES MEMBRES DE CET ESPACE }\end{array}$ \\
\hline
\end{tabular}

Il est important de rappeler que conformément à la démarche explicitée dans le cadre de la SPA, GALATANU, pour les besoins de son étude, (exposée ci-dessus), a recours ici à une valeur présente dans le noyau de Francophonie préalablement déterminée : appartenir à la communauté $X$ donc parler français. C'est donc à partir de ce noyau que sont déclinés les blocs d'argumentation interne (voir ci-dessus). Ainsi, cette analyse rend compte de plusieurs déploiements possibles générés par les blocs d'argumentation 
interne. En approfondissant l'étude, GALATANU réalise un autre déploiement à partir, cette fois-ci, du stéréotype :

PARLER LE FRANÇAIS DC PARTAGER

Nous obtenons alors par déploiement le stéréotype suivant :

"Savoir parler français DC former une communauté linguistique

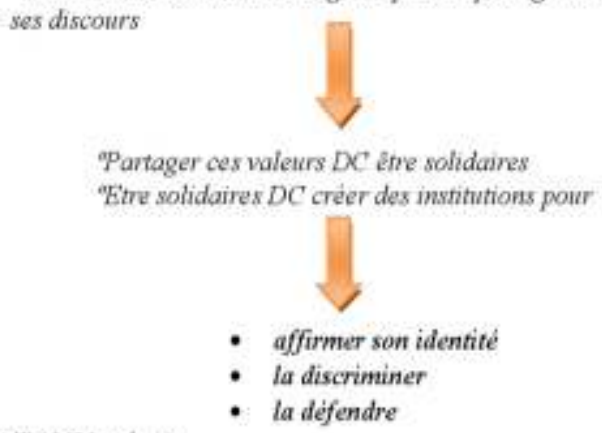

À partir des possibles argumentatifs convoqués dans le discours des apprenants moldaves ${ }^{10}$, nous proposons d'approfondir notre étude en donnant les déploiements générés par les blocs d'argumentation interne obtenus à partir du noyau de Francophonie ${ }^{11}$. Nous obtenons donc le stéréotype suivant :

Novau : PARLER FRANCAIS donc UTILISER LA LANGUE

Stéréotype : Utiliser la langue donc approfondir les connaissances linguistiques

Approfondir les connaissances linguistiques donc effectuer un stage

Effectuer un stage donc réussite

Réussite donc gain

Gain donc réussite professionnelle

Réussite professionnelle donc réalisation de soi

\section{Réussite sociale Réalisation de soi Réalisation d'un projet}

Ce déploiement argumentatif, nous permet d'obtenir une représentation de la Francophonie véhiculée par l'ensemble des étudiants dans leurs discours. Nous voyons apparaître la notion d'utilité associée systématiquement à la langue française. Cette association génère dans le noyau de la Francophonie une valeur liée à l'action (utilité/ 
faire) dont la finalité se traduit par une réussite (réalisation sociale, personnelle, professionnelle).

Conclusion

La SPA fournit ainsi à la signification lexicale l'existence d'un noyau contenant des informations stables sur lesquelles peuvent s'insérer des enchaînements argumentatifs, décrits en termes de blocs d'argumentations internes, les stéréotypes; et externes, appelés possibles argumentatifs.

Cette approche de la SPA permet au niveau sémantique de mettre en exergue des mécanismes explicatifs et surtout de rendre compte de la capacité du discours à véhiculer des systèmes de valeur et à les transformer.

Dans l'approche linguistique des valeurs que nous proposons, le modèle de description sémantique (SPA), modèle s'inscrivant dans le courant de la sémantique argumentative, holistique, permet d'entrevoir et d'observer, d'une part, les évolutions des significations des entités lexicales se rapportant aux valeurs sociales et, d'autre part, de déployer les incidences de ces évolutions sur le fonctionnement du système de valeurs dans son ensemble. Autrement dit, la parole apparaît non seulement comme l'interstice de la manifestation des systèmes de valeurs et d'influence, mais également, comme un lieu de jaillissement de nouvelles valeurs et de nouveaux systèmes de penser de la société et l'homme.

\section{BIBLIOGRAPHIE}

AMOSSY R. (1994) : «Stéréotypie et argumentation », in Le stéréotype -Crise et transformations, Presses Universitaires de Caen, pp. 47-61.

AMOSSY R. et HERSCHBERG PIERROT A. (1997) : Stéréotypes et clichés. Langue, discours, société, Paris, Nathan.

ANSCOMBRE JC., DUCROT O. (1983) : L'argumentation dans la langue, Bruxelles, Pierre Mardaga.

ANSCOMBRE JC. et DUCROT O. (1988) : L'Argumentation dans la langue, Liège Bruxelles, Pierre Mardaga, $3^{\text {ième }}$ édition.

CAREL M. et DUCROT O. (1999) : « Le problème du paradoxe dans une sémantique argumentative ", in Langue Française n¹23, sept. 1999, pp. 6-26.

GALATANU O. (1999a) : « Le Phénomène sémantico-discursif de déconstruction-reconstruction des topoï dans une sémantique argumentative intégrée », in Galatanu, O., Gouvard, J. - M (éd.), Langue Française, $n^{\circ} 123$, Sémantique du stéréotype, pp. 41-51

GALATANU O. (1999b) : « Argumentation et analyse du discours », in Gambier, Y, Suomela-Salmi, E. (éd.), Jalons 2, Turku, Université de Turku, pp. 41-54.

GALATANU O. (1999c) : « Signification, sens et construction discursive de soi et du monde », in BARBIER JM. et GALATANU O. (dir.), Signification, Sens, formation, PUF, pp. 25-43. 
GALATANU O. (2000a) : « La Reconstruction du système de valeurs convoquées et évoquées dans le discours médiatique ", in Actes du XXIIe congrès international de linguistique et philologie romanes, Bruxelles, 23-29 juillet 1998, Max Niemeyer Verlag, vol. VII, pp. 251-258.

GALATANU O. (2000b) : « Langue, discours et systèmes de valeurs », in Suomela-Salmi, E. (Ed.), Curiosités linguistiques, Université de Turku, pp. 80-102.

GALATANU O. (2006) : « La dimension axiologique de la dénomination », in RIEGEL M., SCHNEDECKER C., SWIGGERS P. et TAMBA I. (éds.), Aux carrefours du sens. Hommages offerts à KLEIBER G., Louvain, Peeters.

GALATANU O. (2007a) : « Pour une approche sémantico-discursive du stéréotypage à l'interface de la sémantique théorique et de l'analyse du discours ", in BOYER H. (éd.), Stéréotypage, stéréotypes : fonctionnements ordinaires et mises en scène, Tome 4 Langue(s), Discours, L'Harmattan, Paris, pp. 89-100.

GALATANU O. (2007b) : " Sémantique des possibles argumentatifs et axiologisation discursive », in BOUCHARD D., EVRARD J. (éds.), Représentation du sens linguistique II, De Boeck-Duculot, Louvain-la-Meuve, pp. 313-325.

GALATANU O. (2008a) : « La construction discursive de la Francophonie : sens, valeurs et images identitaires ", in Performances et objets culturels, XI e colloque de Sémiotique de la francophonie, Congrès de l'ACFAS, 15-18 mai 2006, pp. 1-12.

GALATANU O. (2008b) : « La construction discursive des représentations de la langue française et de la francophonie chez les futurs enseignants du FLE en Asie-Pacifiques », in Actes du Premier Congrès Régional Asie-Pacifique, Le français, la francophonie et la francophilie en Asie-Pacifique : spécificités et interrogations, 27 avril-2 mai 2006.

JAHODA M. (1964) : «Stéréotype », A Dictionary of the Social Sciences, London, Tavistock, Publications.

KLEIBER G. (1999a) : « Les proverbes : des dénominations d'un type «très très spécial » ", in Langue française, vol. 123, pp. 52-59.

KLEIBER G. (1999b) : Problèmes de sémantique. La polysémie en question, Lille, Presses Universitaires du Septentrion.

MARIE V. (2003-2004) : "Grâce : étude du noyau et de la déclinaison des possibles argumentatifs », Dossier de DEA, sous la direction de GALATANU O., pp. 2-11.

MORFAUX JM. (1980) : « Stéréotype », Vocabulaire de la philosophie et des sciences humaines, Colin. SPERBER D. (1996) : La contagion des idées, Paris, éditions Odile Jacob.

\section{NOTES}

1. Le stéréotype dans Le Grand Dictionnaire Universel du XIXe de Larousse est défini comme suit : « imprimé avec des planches dont les caractères ne sont pas mobiles, et que l'on observe pour de nouveaux tirages ». " La stéréotypie, quant à elle, désigne soit l'art de stéréotyper, soit l'atelier où l'on stéréotype, plutôt dénommé « clicherie » ». 2. JAHODA M. donne la définition suivante du stéréotype : « croyances concernant les classes d'individus, des groupes ou des objets qui sont préconçues, c'est-à-dire qui ne relèvent pas d'une appréciation neuve de chaque phénomène mais d'habitudes de jugement et d'attentes routinières [...]. Un stéréotype est une croyance qui ne se donne 
pas comme une hypothèse confirmée par des preuves mais est plutôt considérée, entièrement ou partiellement à tort, comme un fait établi », (JAHODA M. 1964:694, "Stéréotype », A Dictionary of the Social Sciences, London, Tavistock Publications, 1964). 3. MORFAUX JM. : « clichés, images préconçues et figées, sommaires et tranchées, des choses et des êtres que se fait l'individu sous l'influence de son milieu social (famille, entourage, études, profession, fréquentations, médias de masse, etc.) et qui déterminent à un plus ou moins grand degré nos manières de penser, de sentir et d'agir », (MORFAUX JM. 1980 : 34, «Stéréotype », Vocabulaire de la philosophie et des sciences humaines, Colin).

4. Le « cinétisme » est entendu par GALATANU, dans le cadre de la SPA, comme une incidence linguistique du discours ou une transformation lexicale intervenant dans le processus holistique et associatif.

5. Pour la « lecture de forme logique » voir SPERBER, 1996.

6. Ce principe renvoie à un article de GALATANU qui présentait les stéréotypes comme faisant partie du noyau de la signification lexicale. Cette approche se présente comme une étape antérieure à la SPA.

7. L'étude du mot « grâce » a été réalisée dans le cadre d'un dossier en DEA Sciences du Langage, à l'université de Nantes, MARIE V (2003-2004) : « Etude du noyau et de la déclinaison des possibles argumentatifs », Dossier de DEA, sous la direction de GALATANU 0., pp. 2-11.

8. S : représente le sujet communiquant et $\mathbf{A}$ le destinataire.

9. Définition proposée par DENIAU, $1983: 1$. Sens linguistique qui renvoie au fait d'être francophone, de parler habituellement ou fréquemment le français, soit comme langue maternelle, soit comme langue officielle, soit comme langue véhiculaire. 2. Sens géographique qui renvoie aux peuples et aux hommes qui ont pour langue maternelle, officielle, courante ou administrative le français. 3. Sens spirituel qui renvoie au sentiment d'appartenir à la même communauté. 4. Sens institutionnel qui renvoie à l'ensemble des institutions et organismes qui œuvrent pour la francophonie.

10. Le discours des apprenants moldaves se présente sous forme de questionnaires recueillis dans le cadre de la thèse afin de constituer un corpus.

11. Notre méthode d'analyse s'appuie sur celle de GALATANU développée dans la partie 2. Analyse du concept de «Francophonie " dans les discours lexicographiques et dans le processus de dénomination institutionnelle.

\section{RÉSUMÉS}

Cet article se donne pour tâche d'illustrer le modèle théorique de la SPA (Sémantique des Possibles Argumentatifs) proposé et développé par GALATANU. Cette théorie se présente comme un modèle de description de la signification lexicale susceptible de rendre compte aussi bien des représentations du monde perçu et «modélisé » par la langue que du «potentiel argumentatif » des mots, potentiel que l'environnement pragmatique de la phase énoncée et/ou l'environnement pragmatique (le contexte du discours) peuvent activer, voire renforcer ou, au contraire, affaiblir, voire neutraliser ou même intervertir. Cette théorie s'inscrit dans la filiation 
de la sémantique argumentative (ANSCOMBRE, DUCROT, 1983, 1995 ; DUCROT, CAREL, 1999) et d'autre part, dans celle de la recherche sur les stéréotypes linguistiques (AMOSSY, 1994 ; KLEIBER, 1999a, b). Dans l'espace de cette communication nous nous proposons de présenter et d'appliquer ce modèle aux notions «francophile, francophilie, francophone, francophonie » qui rendra compte des déploiements argumentatifs en analysant l'évolution des définitions lexicographiques étudiées en termes de noyau et de stéréotypes.

\section{INDEX}

Mots-clés : stéréotypes, stéréophagie, signification lexicale, SPA, sémantique argumentative, linguistique, pragmatique, analyse du discours, noyau, francophonie, fittérature francophone, francophile

\section{AUTEUR}

\section{VIRGINIE MARIE}

Docteur en Sciences du langage 